\title{
Changes in Cell Distribution during Mouse Secondary Palate Closure in Vivo and in Vitro
}

\author{
I. Epithelial Cells
}

\author{
LINDA L. BRINKLEY
}

Department of Anatomy and Cell Biology, Medical School, University of Michigan, Ann Arbor, Michigan 48109

Received November 4, 1982; accepted in revised form September 19, 1983

\begin{abstract}
The distribution of epithelial cells around the perimeter of mouse secondary palatal shelves was observed before and after shelf reorientation in vivo and in vitro. Changes in shelf perimeter, cells per micrometer, and cell layering were measured for each of three shelf regions: anterior and posterior presumptive hard and presumptive soft palate at developmental stages which were 30,24 , and $18 \mathrm{hr}$ prior to expected in vivo elevation, after in vivo elevation, and during the course of in vitro elevation. Pronounced incresses in numerical cell density and cell layering accompanying shelf reorientation were noted in the superior nasal and mid-oral portions of the shelf perimeter in all three shelf regions with greatest changes noted in the posterior hard palate region. These changes were not attributable to cell division or to perimeter changes. The localized nature of the changes in cell distribution suggest that the underlying mechanisms may also be localized.
\end{abstract}

\section{INTRODUCTION}

The reorientation of the secondary palatal shelves from a vertical position on either side of the tongue to a superior, horizontal position is acknowledged to result in large part from the action of an internal force within the shelves themselves (Walker and Fraser, 1956). By its intrinsic nature, such a force must arise from the independent or combined action of various components of the shelves. Since palatal shelf elevation can take place in vitro in fetal mouse heads after removal of the tongue and brain, a dissection which precludes vascular or neural activity (Brinkley, et al., 1975, 1978), it seems reasonable to assume that internal shelf force does not require the direct action of these elements. Intrinsic shelf force must arise from the activities and interplay of the shelves' basic structural components: mesenchymal cells, extracellular molecules, and surrounding epithelium.

Knowledge of whether or not alterations in the spatial patterning of these shelf components occur concommitantly with shelf reorientation would provide important clues to the nature of the underlying mechanism. Yet only anecdotal information is available on changes in patterns of cell distribution in the shelves during elevation. Shelf epithelium has been reported to increase in thickness and to undergo local changes in cell shape following elevation (Pourtois, 1972; Ferguson, 1977; Babiarz et al., 1979). Mesenchymal cells have been observed to disperse just prior to shelf elevation (Kochhar and Johnson, 1965; Sweney and Shapiro, 1970; Ferguson, 1977;
Brinkley, 1980). Despite indications that major shifts in both epithelial and mesenchymal cell patterning are probably taking place during the course of shelf movement, no systematic quantitative studies of cell patterning over the course of palatal shelf elevation have been done.

Changes in cell distribution could be transient over the course of shelf movement and thus might go undetected if shelves were examined only before and after in vivo elevation. For optimum monitoring of cell displacements it is necessary to have a means of following changes in the shelves during the dynamic phases of elevation. Our in vitro system supports palatal shelf elevation with good tissue vitality. This permits shelf movement to be monitored beginning as early as $11 / 2$ days prior to expected in vivo palate closure (Brinkley et al., 1975; Lewis et al, 1980). Thus, changes in cell patterning can be followed over the course of shelf movement.

The present study reports changes in epithelial cell distribution associated with palatal shelf movement. Specific regional patterns of epithelial cell displacements were found which correlate with shelf elevation and are not attributable to cell division.

\section{MATERIALS AND METHODS}

\section{Animals}

A random-bred CD-1 mouse strain was used for all experiments. Animals were maintained in quarters with 
a dark cycle from 8 PM to 6:30 AM and fed Purina mouse chow and water ad libitum. Fertilization was assumed to occur between midnight and $2 \mathrm{AM}$ of the morning the vaginal plug was found. Palate closure, that is, shelf reorientation and initial adhesion, normally occurs by Day 14.5 in these mice.

Animals were sacrificed by cervical dislocation and the fetuses removed under sterile conditions at four different developmental times selected to span the course of secondary palate formation. Chronological age as a measure of development is imprecise, thus fetuses were grouped according to developmental stage. These stages were defined by both crown-rump length (CRL) and a morphological rating (MR) based on the developmental state of fore- and hindlimbs, ears, eyelids, and hair follicles using the system of Walker and Crain (1960). Four groups were selected by these criteria: (1) CRL 9.0-9.5 mm, MR 3-4; (2) CRL 10.0-10.5 mm, MR 5-6; (3) CRL 11.0-11.5, MR 7-8; (4) CRL 12.5-13.0, MR 12-14. These groups were approximately 30,24 , and $18 \mathrm{hr}$ prior to and at the time of expected in vivo palate closure, respectively. They will subsequently be referred to by MR.

Immediately after the fetuses were obtained and staged, the heads were removed. Some intact (I) heads from each group were fixed immediately in phosphatebuffered formalin. In the remainder the brain and tongue (BT) were removed in the following manner. A single circumferential cut was made just above the eyes and the upper portion of the cranium and the brain removed. The tongue was removed by excising the floor of the oral cavity while leaving the mandible in place as previously described (Brinkley et al, 1975). The opening created by tongue removal allowed for circulation of culture medium into and out of the oral cavity. Some BT heads were then fixed to serve as time zero $\left(T_{0}\right)$, unincubated controls and the rest were hung in the gassed, circulating culture system previously described (Lewis et al., 1980).

Palatal shelves have anatomically identifiable regions which behave in a region-specific manner during shelf elevation (Brinkley and Vickerman, 1979, 1982). Anatomical landmarks used to identify the regions were those of Diewert (1978): (1) anterior hard palate: nasal septum, vomeronasal organs, and nasal cavity; (2) posterior hard palate: posterior aspects of nasal cavity or immediately posterior to it, maxillary and mandibular tooth germs; and (3) soft palate: cranial structures and the cranial base.

The anterior hard palatal and soft palatal regions of all three ages of $T_{0}(\mathrm{BT})$ as well as the posterior region of MR 3 and 5 specimens were unelevated. MR 7 specimens occasionally showed some reorientation of the nasal surface from the vertical after tongue removal (Fig. 8), but not of a magnitude to be termed partial elevation.

\section{Culture, Labeling, and Histological Techniques}

The culture medium consisted of BGJb medium (GIBCO) with $10 \%$ fetal bovine serum (KC Biologicals), supplemented to a final concentration of $8 \mathrm{~m} M$ glutamine and containing $50 \mu \mathrm{g} / \mathrm{ml}$ gentamicin (Schering Corp.). The medium was constantly circulated, gassed with $95 \%$ $\mathrm{O}_{2}-5 \% \mathrm{CO}_{2}$ using silicone copolymer hollow-fiber devices to maintain a $\mathrm{pO}_{2}$ of $600 \pm 30 \mathrm{Torr}$, and kept at $34^{\circ} \mathrm{C}$. The unique combination of a high partial pressure of oxygen in the medium combined with constant medium circulation and local stirring around and within the oral cavity of the partially dissected explants has previously been shown to provide good tissue viability with no unusual areas of necrosis after incubation times of $24 \mathrm{hr}$ (Lewis et al., 1980). Specific details of the dissection procedures and culture system are described in Brinkley et al. (1975) and Lewis et al. (1980), respectively.

Specimens were incubated 6,12 , or $18 \mathrm{hr}$. To determine the influence of cell division on epithelial cell patterning in vitro, $\left[\right.$ methyl $\left.{ }^{3} \mathrm{H}\right]$ thymidine (specific activity $40-60$ $\mathrm{Ci} / \mathrm{mmole}$; New England Nuclear) was present in the culture medium at a final concentration of $20 \mu \mathrm{Ci} / \mathrm{ml}$ during the entire 6-hr incubation period. The same concentration of isotope was also added for the last $6 \mathrm{hr}$ of both the 12- and 18-hr incubation periods. At the end of the incubation periods specimens were rinsed in three changes of medium with no isotope, fixed for $2-4 \mathrm{hr}$ in phosphate-buffered formalin, dehydrated through an ethanol series, and embedded in glycol methacrylate.

Three micrometer, serial, cross-sections were taken through the anterior and posterior presumptive hard palate and the presumptive soft palate. Pairs of contiguous sections were separated and placed on two different gelatin-coated slides. One set was used for autoradiography and dipped in NTB-2 photographic emulsion (Kodak), placed in light-tight boxes and exposed for 2 weeks at $0^{\circ} \mathrm{C}$. The slides were then developed using D-19 developer (Kodak) and left unstained. The second set was stained with toluidine blue.

\section{Data Collection}

Using a microscope and drawing tube, the stained sections from each of the three shelf regions were traced and the location of each epithelial cell nucleus marked. The image of the unstained, autoradiographed adjacent section was then projected on this tracing and the location of each labeled nucleus was also marked. Labeled nuclei were intensely black and easily distinguishable. All labeled nuclei were densely covered with blackened silver grains. Background labeling was approximately five grains per square centimeter.

The shelf perimeters of the traced cross sections were delimited as previously described (Brinkley and Vickerman, 1982). The method is shown diagrammatically 


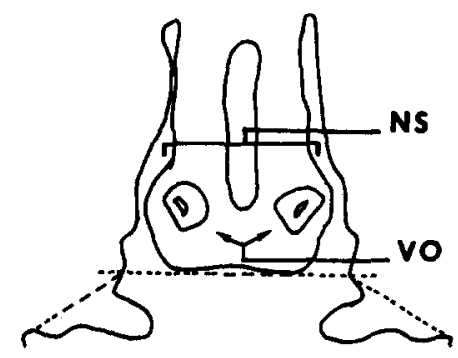

(nc)

ANTERIOR

HARD PALATE

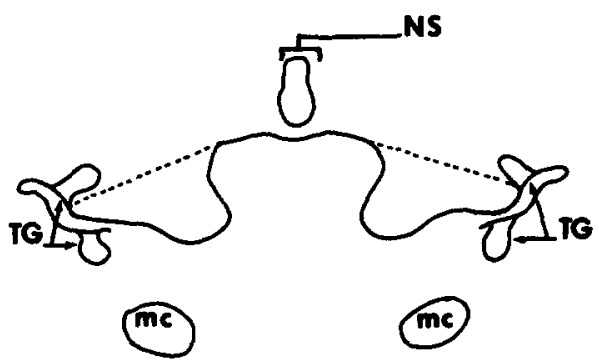

POSTERIOR

HARD PALATE

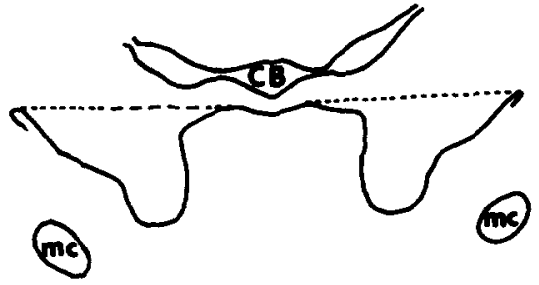

SOFT PALATE

Fig. 1. Schematic drawing of method used to delimit shelf boundaries. For the anterior shelf a horizontal line in register with the inferior surface of the nasal septum (NS) is drawn. Lateral shelf boundaries are then determined by drawing lines from each edge of the oral cavity to the point of intersection with that horizontal line. Lateral borders for the posterior hard and soft palatal shelf regions are determined by drawing a line from the medial edge of the tooth germs (TG), if present, or the edge of the oral cavity if none are present, tangent to the curve of the roof of the oral cavity. The first point of intersection of this line with the roof of the primitive oral cavity was then used as the most superior point of the shelf. The vomeronasal organs (VO), Meckel's cartilage (MC), and the cranial base (CB) are shown for orientation.

in Fig. 1. The perimeters were measured using a planimeter and divided into six equal segments beginning at the upper nasal boundary of the shelf. Thus segment 1 was on the most superior, nasal surface of the shelf, segment 6 at the most lateral extent of the oral side of the shelf (Fig. 2). For each segment the number of cells $(\mathrm{C} / \mu \mathrm{m})$ and the number of labeled cells $(\mathrm{LC} / \mu \mathrm{m})$ per linear micrometer were calculated and the maximum number of cell layers (CL) recorded. The shapes of the cells in each segment, whether squamous, cuboidal, or columnar were also noted.

\section{Data Analysis}

The time-consuming nature of the data collection process precluded large sample sizes, thus groups were selected to provide as much sampling independence as possible. For each combination of age and dissection (I or BT) or age and incubation time $\left(T_{0}, 6,12,18 \mathrm{hr}\right)$ one

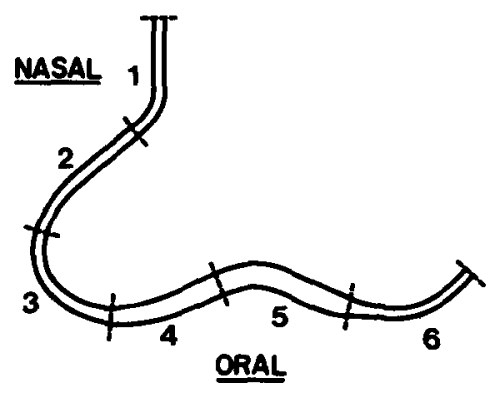

FIG. 2. Segmentation of the shelf perimeter. The epithelial perimeter of each shelf was traced, measured and divided into six equal segments numbered from one on the superior nasal surface to six on the lateral oral side. The number of cells per micrometer $(\mathrm{C} / \mu \mathrm{m})$, whether labeled or unlabeled, and the maximum number of cell layers, were then noted. fetus was selected from each litter and one shelf of that fetus analyzed, thus sample sizes $(n)$, are the numbers of individuals. A two-way analysis of variance (Scheffé, 1959) was used to analyze changes in shelf perimeter by region as a function of dissection (I vs BT) or of incubation times. Differences were found to be significant when $P<0.05$.

A four-factor mixed model analysis of variance (Scheffé, 1959) was used to test for differences in means between the levels of perimeter segments (1-6), age (MR $3,5,7$, or 12), culture time $\left(T_{0}, 6,12,18 \mathrm{hr}\right)$, and shelf position (unelevated (U), partially elevated (PE), elevated (E), elevated and adhered (EA)). These analyses tested the null hypotheses that there were no differences in the means of the number of cells per micrometer, number of cell layers, or labeling ratios in the six different perimeter segments as a function of each of these four factors. A null hypothesis was found to be invalid when $P<0.05$.

With small sample sizes it requires a reasonably large effect to show that differences are significant. As reported below, such effects were observed.

\section{RESULTS}

Changes in shelf perimeter $\mathrm{C} / \mu \mathrm{m}$ and $\mathrm{CL}$ were measured in fetuses at three stages prior to in vivo elevation: MR 3, 5, and 7 and at MR 12 after in vivo elevation had occurred. Shelf perimeters of MR 3, 5, and 7 specimens were also measured after 6,12 , and $18 \mathrm{hr}$ in vitro.

\section{Changes in Shelf Perimeter}

In vivo. The perimeters of anterior and posterior hard palatal and soft palatal shelf regions from specimens ranging in stage from MR 3 to 12 were measured in 
intact heads and those with the brain and tongue removed (Fig. 3). For each shelf region and stage, no differences between I and BT were noted. In addition, the perimeters of all three shelf regions were similar in both I and BT unelevated states regardless of stage.
Despite this, the cross-sectional shape of all three shelf regions of MR 3 specimens was somewhat different from that seen at MR 5 and 7, particularly in the posterior and soft shelf regions (Fig. 8).

After in vivo elevation at MR 12 (shown as dotted

ANTERIOR

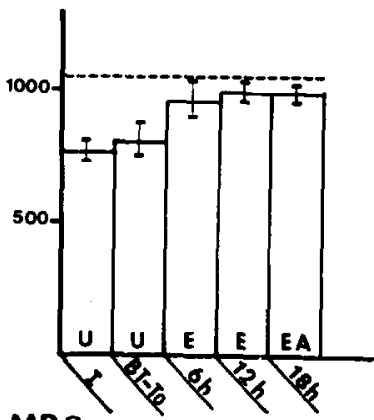

MR 3

\section{POSTERIOR}

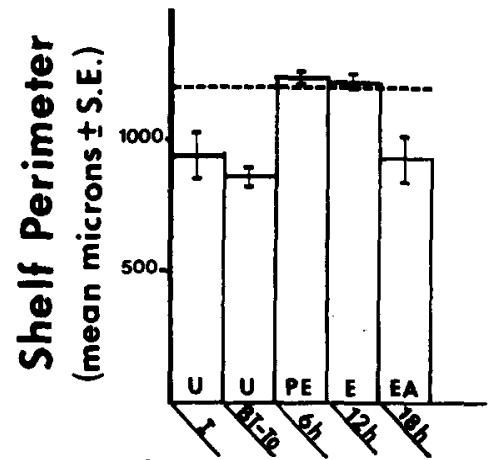

MR 3

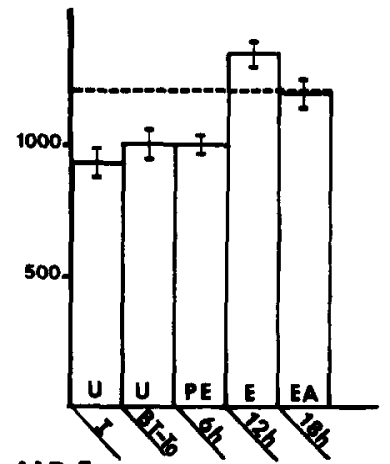

MR 5

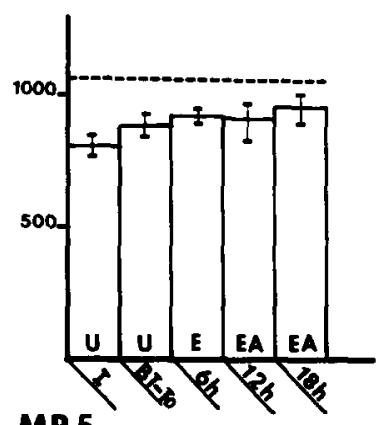

MR 5

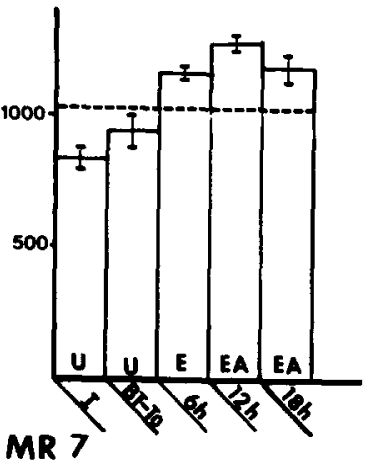

MR 7

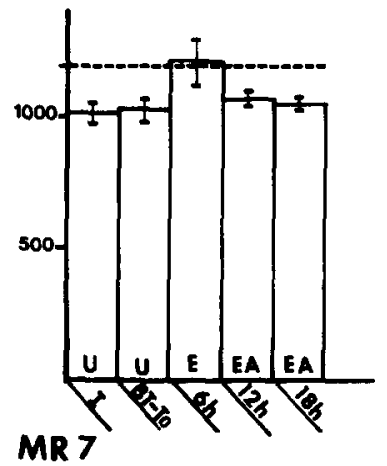

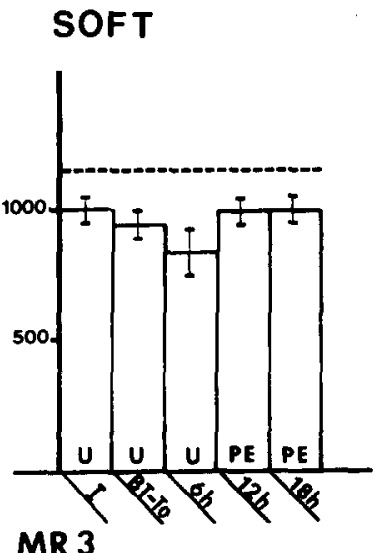
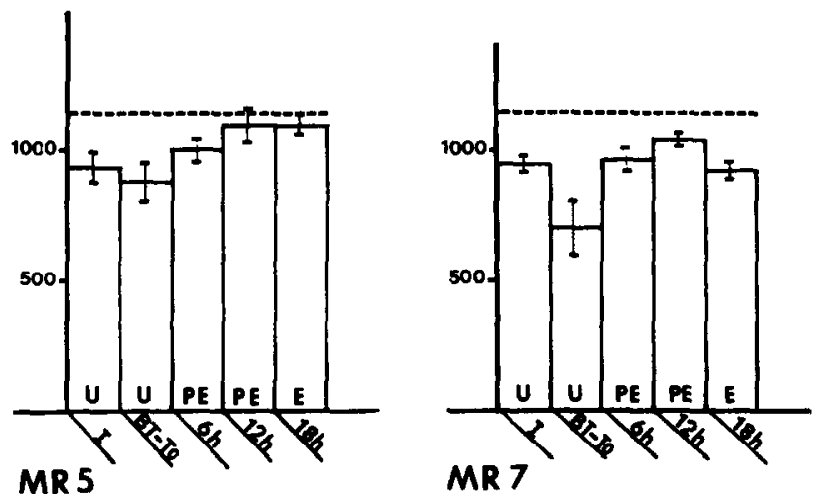

MR 7

FIG. 3. Changes in shelf perimeter during in vivo and in vitro shelf reorientation. The dotted lines across each graph represent the perimeter values for morphologic rating (MR) 12 in vivo reoriented shelves $(n=7)$. The perimeters of all three shelf regions were similar in the unelevated state, regardless of age. The sample sizes for MR 3 were $\mathrm{I}, n=4 ; \mathrm{BT}, n=5 ; 6 \mathrm{hr}, n=4 ; 12 \mathrm{hr}, n=5 ; 18 \mathrm{hr}, n=5$. Those for MR 5 were I, $n=8$; BT, $n=7 ; 6 \mathrm{hr}, n=8 ; 12 \mathrm{hr}, n=4 ; 18 \mathrm{hr}, n=6$, and for MR 7, I, $n=12$; BT, $n=6 ; 6 \mathrm{hr}, n=4 ; 12 \mathrm{hr}, n=6 ; 18 \mathrm{hr}, n=6$. I, intact; BT, brain and tongue removed; U, unelevated; PE, partially elevated; E, elevated; EA, elevated and adhered. 
lines on Fig. 3) the perimeter of all three shelf regions had increased significantly by about $20 \%$ from that seen in unelevated, I or BT, $T_{0}$, specimens of MR 3,5 , or 7 .

In vitro. The ability of the palatal shelf to reorient in vitro was acquired in a rostral to caudal sequence. The anterior region reoriented before the posterior region and the latter did so before the soft palatal shelf region. The sequence was observed in specimens of all three stages studied in vitro. At MR 3, the anterior shelf was able to reorient within $6 \mathrm{hr}$ of incubation. The posterior shelf required twice that long, and the soft region only partially reoriented within the 18 -hr incubation period. By MR 5 the entire shelf was able to fully reorient in vitro. However, the rate of reorientation of the posterior region was more rapid for MR 5 than for MR 3 specimens. By MR 7 both anterior and posterior regions were able to elevate in vitro within $6 \mathrm{hr}$, whereas the soft shelf region still required $18 \mathrm{hr}$ of incubation as did MR 5 specimens.

The MR 3 and 7 anterior shelves increased their perimeters significantly at elevation, and did not change further after adhesion occurred, but MR 5 shelves showed no such increase. The perimeters of MR 3 shelves attained lengths equivalent to that seen in MR 12 in vivo elevated shelves, while those of MR 7 were somewhat greater than that of in vivo elevated shelves.

An increase in perimeter of posterior shelf coincident with reorientation was followed by a perimeter decrease after adhesion in specimens of all three stages. Elevated posterior shelves of all three stages had shelf perimeters equivalent to those of the MR 12 in vivo elevated posterior shelves.

No changes in perimeter were found in partially elevated soft palate of MR 3 specimens and the perimeters remained significantly less than those of the same region in in vivo elevated specimens. However, MR 5 and 7 shelves showed perimeter increases at the same shelf position after $12 \mathrm{hr}$ in vitro which were comparable to those of in vivo elevated shelves. A decrease in perimeter was seen in MR 7 shelves after complete elevation.

\section{Changes in Cells/Micrometer}

In vivo. The distribution of $\mathrm{C} / \mu \mathrm{m}$ was statistically uniform across all perimeter segments in the anterior shelf region for all three stages prior to elevation (Figs. 4-6; 8). At MR 12 (Fig. 7), after elevation, a nonuniform distribution was noted: segment 1 was significantly increased whereas the adjoining segments had the same cell density as the unelevated shelves.

In the posterior, MR 3, $T_{0} \mathrm{U}$ specimens (Fig. 4) showed a nonuniform cell distribution with higher cell densities in segments 4 and 5 than in the remainder of the perimeter, whereas cell distribution was more uniform around the posterior regions of MR 5 (Fig. 5) and MR
7 (Fig. 6) specimens. After elevation at MR 12 (Fig. 7), segment 1 again contained the greatest cell density while the rest of the perimeter was uniform.

The soft palate showed uniform distribution of cells at all three ages prior to shelf reorientation. After elevation (Fig. 7), cell distribution was nonuniform, with segments 1,5 , and 6 having greater $\mathrm{C} / \mu \mathrm{m}$.

$\mathrm{CL}$ followed the same patterns as $\mathrm{C} / \mu \mathrm{m}$ for all shelf regions at all four ages studied. MR 3,5 , and 7 specimens averaged two cell layers uniformly distributed around the perimeter of the anterior and posterior shelves, whereas the soft region was usually covered by only one epithelial cell layer. After in vivo elevation at MR 12, all three shelf regions showed an increased layering in segment 1.

In vitro. The pattern of segments showing change after in vitro elevation in anterior, posterior, and soft palatal shelf was similar at all three ages, but the magnitude of changes observed lessened with age (Figs. 46). That is, all regions of MR 3 and 5 shelves exhibited greater local changes in cell density during and after elevation than did those of MR 7.

$M R$ 3. The epithelial perimeter of anterior shelves reoriented after $6 \mathrm{hr}$ incubation increased in $\mathrm{C} / \mu \mathrm{m}$ in segment 1 , the most nasal segment, and in oral segments 4,5 , and 6 when compared to their unelevated counterparts (Fig. 4). No significant changes in CL were observed. Segments 1 and 6 showed an additional increase after $12 \mathrm{hr}$ incubation, but by $18 \mathrm{hr}$ only segment 1 remained more densely populated than the others.

Posterior shelves reoriented more gradually passing through an intermediate partially elevated stage. A gradual change to a nonuniform cell distribution accompanied the reorientation. When partially elevated after $6 \mathrm{hr}$ incubation, only segment 1 showed an increase in $\mathrm{C} / \mu \mathrm{m}$ over that seen in the unelevated condition. At the same time CL increased in segment 1 and decreased in the remaining segments. When the shelves were fully elevated at $12 \mathrm{hr}$, no further changes were noted in $\mathrm{C} /$ $\mu \mathrm{m}$ in segment 1 . However, segments 3,4 , and 5 showed increases in $\mathrm{C} / \mu \mathrm{m}$ from that seen in the same regions of unelevated or 6-hr partially elevated shelves. After $18 \mathrm{hr}$ incubation, adhesion had occurred and a uniform distribution of $\mathrm{C} / \mu \mathrm{m}$ was observed. Cell layering was again at the level of the unelevated condition in segments 3-6.

The soft palate attained partial elevation after $6 \mathrm{hr}$, and no further shelf reorientation occurred with additional incubation. At $6 \mathrm{hr}$, all segments except for segment 2 showed increased $\mathrm{C} / \mu \mathrm{m}$ with the greatest increase seen in segment 6 . No changes in CL were observed to accompany these changes in $\mathrm{C} / \mu \mathrm{m}$. During the subsequent $12 \mathrm{hr}$ of incubation the cell distribution gradually became uniform.

$M R$ 5. The entire perimeter of all three shelf regions 


\section{ANTERIOR}
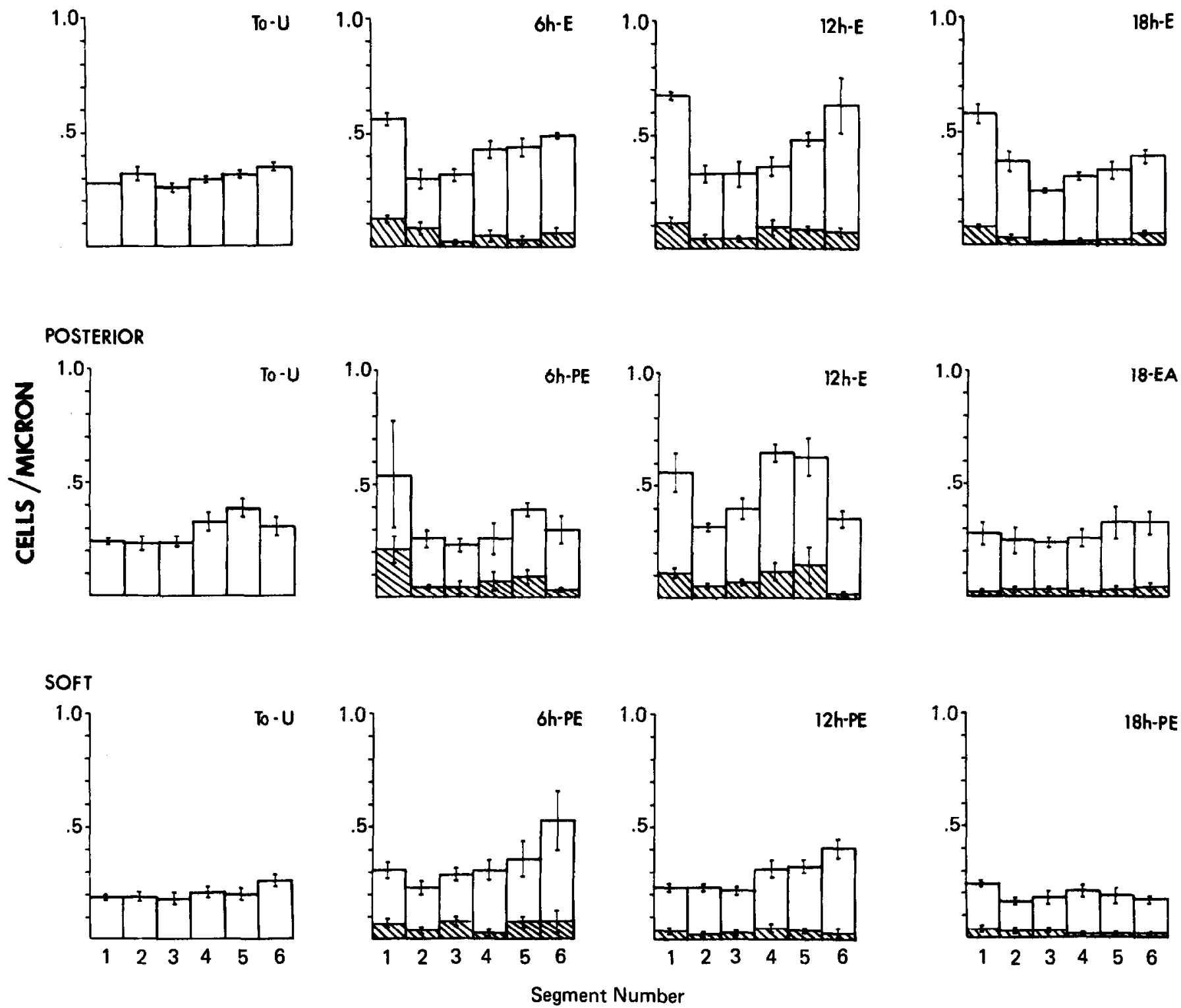

FIG. 4. Changes in $\mathrm{C} / \mu \mathrm{m}$ in MR 3 shelf perimeter segments during the course of in vitro elevation. The ordinate is mean number of cells per linear micrometer \pm standard error of the mean. The cross-hatched portion of each column displays the mean number of labeled cells per linear micrometer \pm standard error of the mean. The segmental patterns of cell distribution observed in the unelevated (U) state was statistically uniform in the anterior and soft palatal shelf regions, whereas posterior unelevated shelves displayed nonuniform distribution with segments 4 and 5 having higher cell densities than the remainder of the perimeter. In the anterior palatal region after elevation (E) at 6,12 , or $18 \mathrm{hr}$, in the posterior palatal region after both partial (PE) and complete elevation, and in the soft palatal region after partial elevation at $6 \mathrm{hr}$, the segmental patterns of cell distribution are significantly different from their respective unelevated conditions, $P<0.05$. Sample sizes were $T_{0}, n=4 ; 6 \mathrm{hr}, n=4 ; 12 \mathrm{hr}, n=8,18 \mathrm{hr}, n=6$. All $T_{0}$ specimens were BT.

showed at least a twofold increase in $\mathrm{C} / \mu \mathrm{m}$ in all segments at $6 \mathrm{hr}$ when compared to their $T_{0}$ unelevated counterparts (Fig. 5). A similar picture was seen in anterior and posterior shelves when CL was examined, but in the soft region layering remained unchanged. In anterior shelves an increase in $\mathrm{C} / \mu \mathrm{m}$ in segment 1 was noted after elevation at $6 \mathrm{hr}$. Adhesion had occurred by $12 \mathrm{hr}$ and the cell density of segment 1 remained high but now, in addition, the density of segment 6 had also increased.

A nonuniform pattern of increased cell density in segments 1,4 , and 5 was also seen in the partially elevated posterior shelves. After elevation at $12 \mathrm{hr}$ the segmental pattern of cell density was similar to that seen in the partially elevated state except that segment 3 had decreased below its value in the partially elevated shelf. 
ANTERIOR
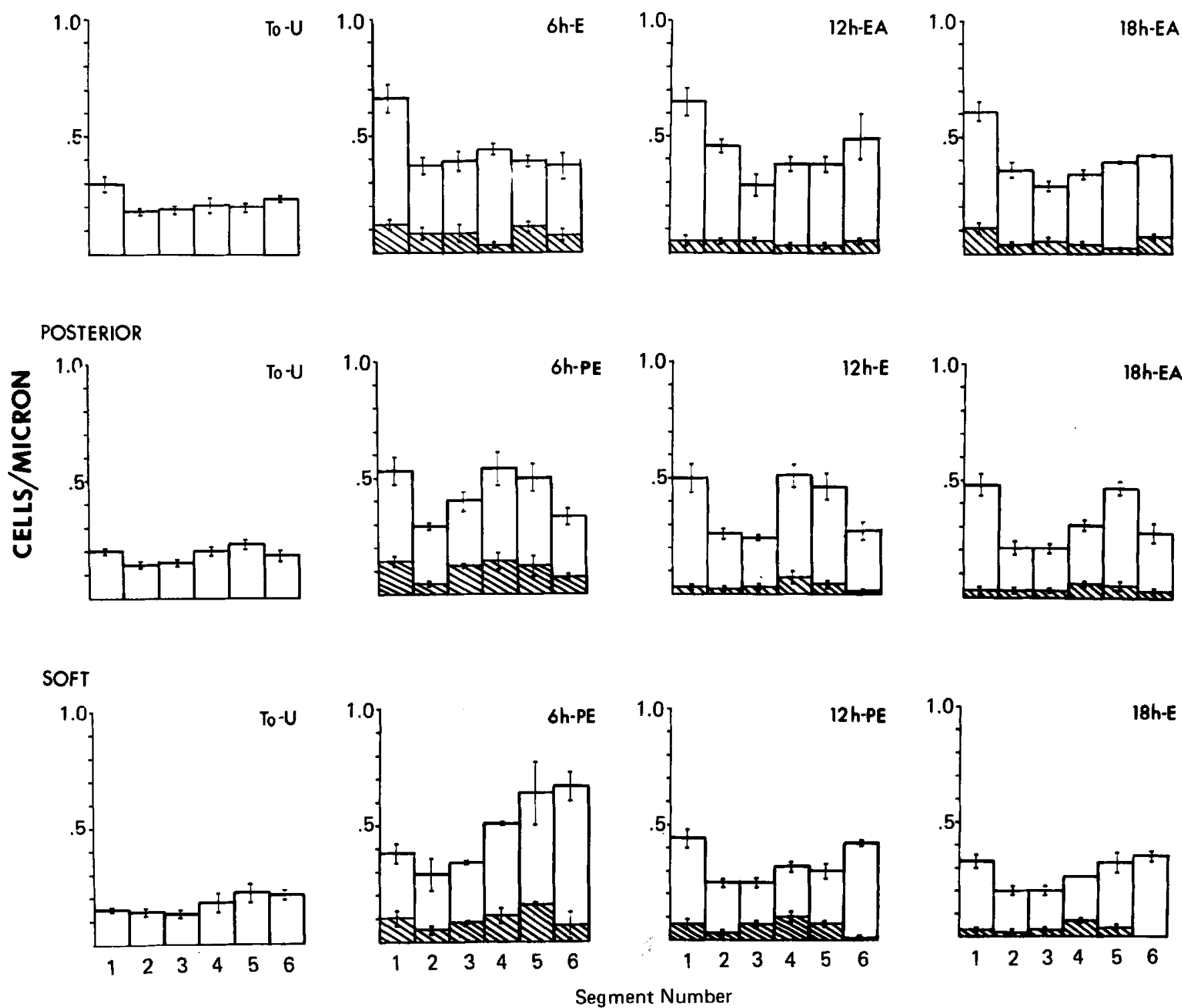

FIG. 5. Changes in $\mathrm{C} / \mu \mathrm{m}$ in MR 5 shelf perimeter segments during the course of in vitro elevation. The ordinate is mean number of cells per linear micrometer \pm standard error of the mean. The cross-hatched portion of each bar displays the mean number of labeled cells per linear micrometer \pm standard error of the mean. At $T_{0}$ unelevated (U), cell distribution was statistically uniform around the entire perimeter in all three shelf regions. The segmental patterns of cell distribution observed in the anterior palatal region after elevation (E) at $6 \mathrm{hr}$ or subsequent adhesion (EA) at 12 or $18 \mathrm{hr}$; in the posterior palatal region after elevation at 6 and $12 \mathrm{hr}$ or adhesion at $18 \mathrm{hr}$; and in the soft palatal region after partial elevation (PE) at 6 and $12 \mathrm{hr}$ or elevation at $18 \mathrm{hr}$ are significantly different from their respective unelevated conditions, $P<0.05$. Sample sizes were: $T_{0}, n=10 ; 6 \mathrm{hr}, n=4 ; 12 \mathrm{hr}, n=6 ; 18 \mathrm{hr}, n=6$. All $T_{0}$ specimens were BT.

The increases in segments 1 and 5 remained even after $18 \mathrm{hr}$ incubation when adhesion had occurred. CL increased overall after partial elevation, with the greatest increases occurring in segments 1,4 , and 5 . No further change in CL was found after elevation.

The segmental pattern of cell distribution in the soft palate again showed greater increases in segments 1,4 , 5 , and 6, with the oral segments (4-6) having the greatest number of $\mathrm{C} / \mu \mathrm{m}$. After $12 \mathrm{hr}$, only segments 1 and 6 remained at increased levels. Elevation occurred by 18 $\mathrm{hr}$, and although the overall magnitude of cell density had decreased, the pattern of cell distribution observed was essentially that seen after partial elevation at $6 \mathrm{hr}$. It was also, virtually, the same pattern of distribution and magnitude seen after in vivo soft palatal shelf elevation (Fig. 7).

$M R$ 7. Only segment 1 showed substantially increased $\mathrm{C} / \mu \mathrm{m}$ in anterior elevated shelves (Fig. 6). The density 
ANTERIOR
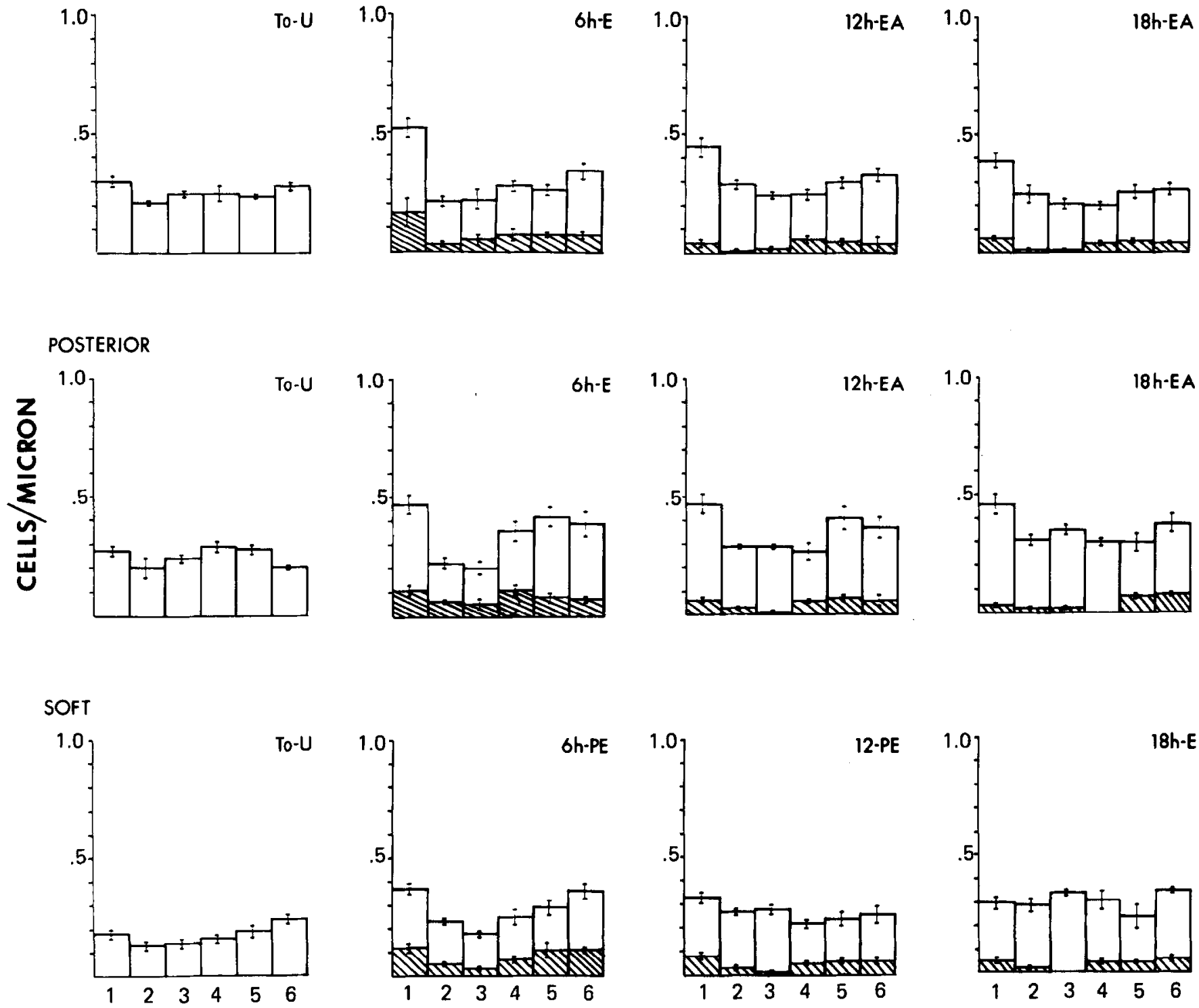

Segment Number

FIG. 6. Changes in $\mathrm{C} / \mu \mathrm{m}$ in MR 7 shelf perimeter segments during the course of in vitro elevation. The ordinate is mean number of cells per linear micrometer \pm standard error of the mean. The cross-hatched portion of each bar displays the mean number of labeled cells per linear micrometer \pm standard error of the mean. At $T_{0}$ unelevated, cell distribution was statistically uniform around the entire perimeter in all three shelf regions. The segmental patterns of cell distribution observed in the anterior palatal region after elevation at $6 \mathrm{hr}$, or adhesion at 12 or $18 \mathrm{hr}$; in the posterior palatal region after elevation at $6 \mathrm{hr}$ or adhesion at 12 or $18 \mathrm{hr}$; and in the soft after partial elevation at $6 \mathrm{hr}$ are significantly different from their respective unelevated conditions, $P<0.05$. Sample sizes were $T_{0}, n=6 ; 6 \mathrm{hr}, n=4 ; 12 \mathrm{hr}, n$ $=6 ; 18 \mathrm{hr}, n=6$.

seen in segment 1 was similar to that found in the same segment after in vivo elevation (Fig. 7). This pattern was evident after elevation at $6 \mathrm{hr}$ incubation and persisted in shelves examined after 12 and $18 \mathrm{hr}$ incubation. No overall increase in $\mathrm{C} / \mu \mathrm{m}$ similar to that seen after elevation of MR 3 and 5 specimens was observed. CL followed the same pattern as $\mathrm{C} / \mu \mathrm{m}$ across all segments.

The pattern of $\mathrm{C} / \mu \mathrm{m}$ in posterior elevated shelves at
$6 \mathrm{hr}$ again showed increases in the most nasal position, segment 1, and also displayed marked increases along the oral side, segments 4,5 , and 6 . These segments gradually decreased in density after adhesion at $12 \mathrm{hr}$ and by $18 \mathrm{hr}$ only segments 1 and 6 showed increased $\mathrm{C} /$ $\mu \mathrm{m}$. CL increased only in segments 5 and 6 .

An overall increase in $\mathrm{C} / \mu \mathrm{m}$ in partially elevated soft palates was noted after $6 \mathrm{hr}$. The greatest increases 


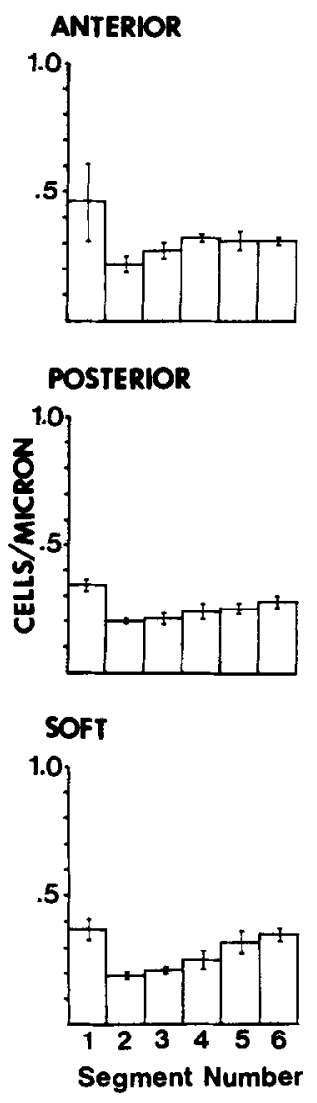

FIG. 7. Segmental distribution of $\mathrm{C} / \mu \mathrm{m}$ ( \pm standard error of the mean) at MR 12 after in vivo reorientation. Anterior and posterior patterns of cell distribution are similar with segment 1 having greater cell density than the remainder. The pattern of cell distribution in the soft palatal region differs from that in the other two regions, in addition to increased cell density in segment 1 , the most lateral oral segments, 5 and 6 , were also increased $(n=5)$.

were seen in segments $1,4,5$, and 6 . After $12 \mathrm{hr}$ incubation the shelves were still partially elevated but a more uniform cell distribution was noted. This uniformity persisted even at elevation after $18 \mathrm{hr}$ incubation. A uniform pattern of distribution of CL was observed before and after partial elevation, but showed an overall increase after elevation.

Schematic diagrams summarizing the distribution of $\mathrm{C} / \mu \mathrm{m}$ and $\mathrm{CL}$ at $T_{0}$ (BT) and after the maximum reorientation attained in vitro are displayed in Fig. 8.

\section{Labeling Ratios}

No correlation between the segmental patterns of labeling ratios, i.e., labeled cells to total cells, seen during in vitro elevation and that of $\mathrm{C} / \mu \mathrm{m}$ and $\mathrm{CL}$ was observed at any of the ages studied (cross-hatched portions of the bars in Figs. 4-6). The average (20\%) and range (17$25 \%$ ) of labeling ratios across the epithelial perimeter were essentially similar for all stages, regions, and both partially and fully elevated shelf positions outside of two local changes. Decreased labeling ratios were noted in the most lateral oral segment, 6 , of MR 5 soft palatal shelves after 12 and $18 \mathrm{hr}$ in vitro. Also at MR 7, after adhesion, all shelf regions showed a pronounced decrease in labeling ratio of the segment or segments which included most of the medial edge (segments 3 and/or 4) where adhesion had occurred.

\section{Changes in Cell Shape}

For all three stages, MR 3, 5, and 7, the epithelial cells of anterior unelevated shelves were predominantly cuboidal in segments 2-5 with a few squamous cells on the upper surface of segments 2-6. Cells of segment 6, the most oral segment, had a more columnar morphology. Immediately after elevation segment 1 cells were stratified columnar with the apical surfaces of the uppermost layer often rounded and bulging as if being squeezed outward. The rest of the perimeter was cuboidal to columnar with some squamous cells on the surface.

Cells of the posterior perimeter were cuboidal in segments 1-3 and more columnar in segments 4-6. After partial elevation, their shapes became more columnar in segment 1 , whereas segments 4,5 , and sometimes part of 6 often became stratified cuboidal with a few squamous cells on the upper surface. This pattern remained after elevation.

In contrast, both unclevated and elevated soft palate had a perimeter composed entirely of cuboidal cells with a few squamous cells on the upper surface.

\section{DISCUSSION}

\section{Segmental Cell Density Patterns are Shelf Region Specific and Stage Dependent}

The present study is the first to demonstrate that quantitative region-specific changes in epithelial cell distribution accompany shelf reorientation. A generally uniform pattern of cell distribution was noted across shelf regions during in vivo development. After in vivo elevation the nasal segments of anterior and posterior shelf and the nasal and lateral oral area of soft palate showed increased cell density. In contrast, striking regional segmental patterns not foreshadowed by the uniform epithelial cell distribution noted prior to shelf reorientation coincided with in vitro elevation. The segmental pattern of cell distribution observed in vitro for the anterior and soft regions was similar to that seen after in vivo elevation. However, the cell distribution around the perimeter of posterior shelves showed local increases, not only in the most nasal segment, as after in vivo elevation, but also along the mid-oral portion of the shelf. 
ANTERIOR
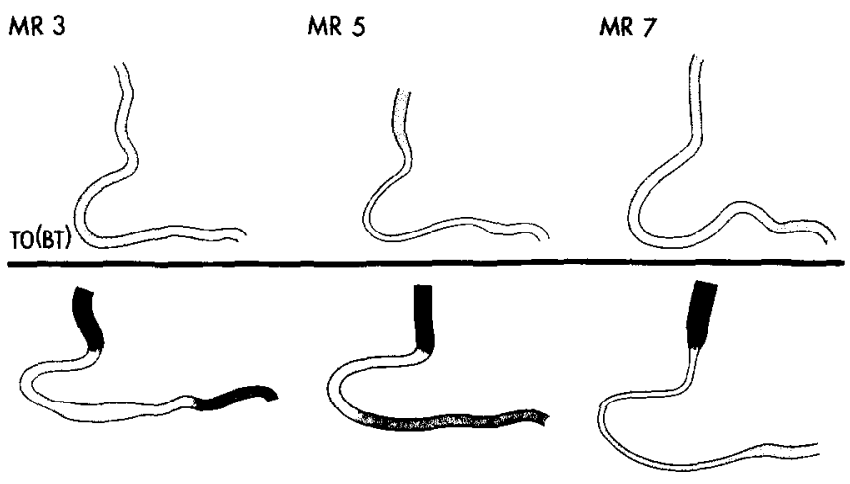

SOFT

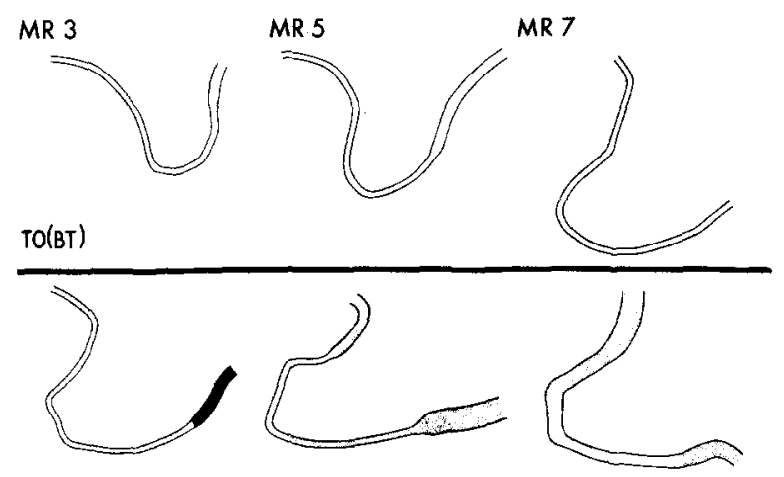

POSTERIOR

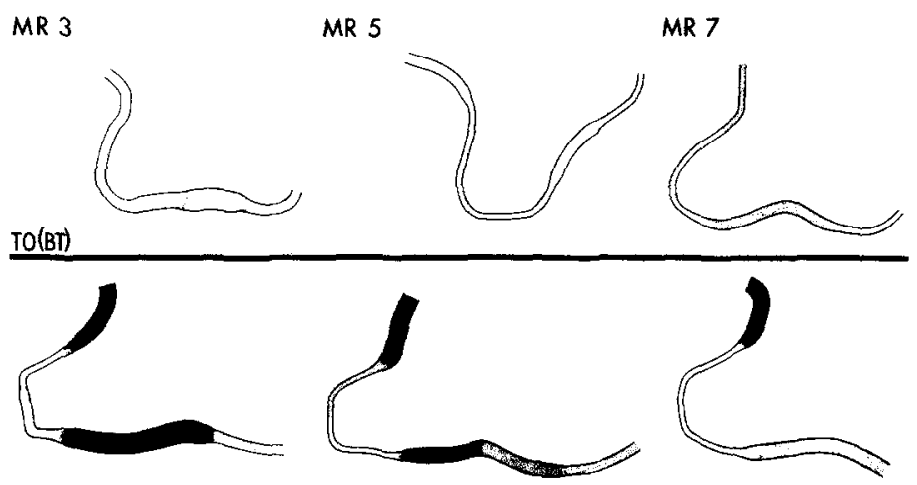

FIG. 8. Schematic summaries of the significant changes in cell density and layering observed at $T_{0}$ (BT) and after in vitro palatal shelf reorientation of MR 3,5, and 7 specimens as compared to the $T_{0}$ (BT) configurations. The top row of each panel illustrates typical shelf form seen after tongue removal and prior to incubation. The second row of each panel illustrates changes seen after the culture interval at which the maximum form change, e.g., elevation or partial elevation in the case of MR 3 soft palate, first occurred (Figs. 4-6). The legend shows the tone pattern and line width codes used.

Despite in vivo and in vitro pattern similarities, the region-specific cell density changes noted during the course of in vitro elevation were of considerably greater magnitude than those seen after in vivo elevation in all shelf regions. Changes in cell distribution seen over the course of in vitro shelf elevation were also observed to lessen with stage, and to occur more rapidly and transiently. For instance, the differential pattern of cell distribution in the posterior shelves became more pronounced after elevation at $12 \mathrm{hr}$ in MR 3, but was obvious after only $6 \mathrm{hr}$ in vitro at MR 5 and although apparent at MR 7 after $6 \mathrm{hr}$ in vitro, was not of the magnitude seen at the earlier stages. The in vitro system allows observation of the reorientation behavior of shelves considerably before they would reorient in vivo. Thus it is possible that the older shelves, MR 7, did in fact show the same magnitude of change, but passed through the more acute phase of cell density changes earlier in the culture period. The present results indicate that following elevation in vivo does not allow a full appreciation of the very localized and significant changes in epithelial cell density which occur during the course of shelf reorientation.

In earlier work we have shown that beginning as much as 36-48 hr prior to in vivo elevation the palatal shelves are able to reorient in vitro when the tongue is removed and that the extent of elevation that is achieved as well as the incubation time required to achieve it is directly correlated with developmental stage (Brinkley et al, 1975; Lewis et al, 1980). The present and past in vitro findings suggest a gradual acquisition of reorientation ability occurs and is fully developed by MR 5, at least $24 \mathrm{hr}$ prior to in vivo reorientation. The stage related alterations in magnitude and speed of change of epithelial cell distribution found to be associated with shelf elevation may be related to the gradual acquisition and/ or maturation of the ability to reorient.

\section{Perimeter Changes and Cell Division Cannot Account for Changes in Cell Density}

No immediate changes in perimeter were observed after tongue removal, and no significant changes in the 
perimeter of any shelf regions were observed to occur with increasing developmental stage prior to in vivo elevation. After in vivo reorientation had occurred, an approximate $20 \%$ increase in perimeter was noted in all shelf regions, although no intermediate stages of perimeter expansion were observed.

This contrasts with our findings on changes in shelf perimeter during in vitro shelf reorientation which supports the idea of regional variation in and acquisition of the ability to expand. Anterior shelves apparently do not need to expand in order to elevate as at only one stage, MR 7, did this region show perimeter expansion after elevation whereas the posterior shelf region showed relatively rapid perimeter expansion correlated with elevation at all three stages. Changes in perimeter of the soft palate which equaled those seen in vivo were also noted after partial elevation in MR 5 and 7 specimens, whereas MR 3 shelves which were unable to fully elevate in vitro had perimeters which remained below the in vivo elevated level. It is interesting that for most regions which were able to fully elevate in vitro, regardless of stage, the final perimeter value attained after elevation and adhesion in vitro was similar to that seen after elevation and adhesion in vivo.

One means by which perimeter expansion may occur is by the addition of new cells. Labeling ratios did not show regional or segmental patterns but rather averaged around $20 \%$ for the perimeter of all shelves. These ratios are similar to those found in vivo in rats by Hudson and Shapiro (1973). If cell size remained relatively constant, as it appeared to, a $20 \%$ perimeter expansion could be accounted for on the basis of cell division alone. This is approximately the increase in perimeter noted in all shelf regions after in vivo elevation in MR 12 specimens and in the posterior of all three ages after in vitro elevation. In addition to this perimeter increase, an overall increase in cell number could also be accounted for by the addition of new cells, but neither shelf perimeter changes nor labeling ratios alone or in combination can account for the distinct nonuniform patterns of epithelial cell distribution observed to occur during shelf elevation.

However, present results do suggest that increase in perimeter is not necessary for anterior shelf elevation, but may be a requirement for elevation of the rest of the palatal shelf. This is also consistent with the suggestion that the anterior shelf tends to move as a whole whereas the remainder of the shelf remodels in a more fluid manner, with a key role in remodeling probably being played by the middle third of the palate, the posterior presumptive hard palate (Brinkley and Vickerman, 1979, 1982). In vivo gradual shelf expansion may not be possible because of the physical constraints imposed on the shelves as they are wedged between the tongue and mandible. It seems possible that in vivo when the caudal two-thirds of the shelf have acquired sufficient expansion ability and spatial relations in the oronasal cavity permit, these shelf regions then rapidly remodel around the tongue, expanding in perimeter as they do so.

\section{Implications of Changes in Cell Distribution}

Changes in epithelial cell distribution, shape, and/or layering associated with shelf elevation were local and reasonably rapid. It seems likely that the segmental and regional changes in cell distribution are therefore under relatively local control. Whether the local and regional changes in epithelial cell density are playing an active role in elevation or are merely passively reflecting other events occurring during shelf elevation is not known. Pourtois (1972) suggested that local changes in epithelial cell adhesivity on the nasal surface and formation of the rugae on the mid-oral surface somehow played a role in shelf reorientation. It appears this could be the case on the nasal surface, although rugae formation does not seem a likely possibility to explain the changes in cell density observed on the mid-oral surface of the posterior and soft regions as no rugae were visible in these areas following in vitro elevation. One way in which the local increases in cell density observed in the nasal and midoral segments of the epithelial perimeter could affect shelf reorientation is by increasing the pressure on the internal mesenchyme and extracellular matrix components and/or by serving to buttress those local regions to resist any displacement of these elements against them. This could in effect direct any movement or displacement of the mesenchymal cells and/or extracellular matrix components toward the medial region. A fluid remodeling of the shelves as seen in the caudal two-thirds of the palate (Brinkley, 1980) might then result.

The author gratefully acknowledges the expert assistance of Kenneth Guire, Statistical Research Laboratory, University of Michigan. This work was supported by NIH-NIDR Grants DE02774 and 5K0400104.

\section{REFERENCES}

BabiarZ, B., WEE, E. and Zlmmerman, E. (1979). Palate morphogenesis. III. Changes in cell shape and orientation during shelf elevation. Teratology 20, 249-278.

BRINKt.EY, I. (1980). In vitro studies of palatal shelf elevation. In "Current Research Trends in Prenatal Craniofacial Development" (R. M. Pratt and R. C. Christiansen, Eds.), pp. 203-220. Elsevier/ North-Holland, New York.

BRINKLEY, L., and VICKERMAN, M. (1979). Elevation of lesioned palatal shelves in vitro. J. Embryol. Exp. Morphol. 54, 229-240.

BRINKLEY, L., and VICKERMAN, M. (1982). Effects of chlorcyclizineinduced alterations of glycosaminoglycans on mouse palatal shelf elevation in vivo and in vitro. J. Embryol Exp. Morphoh 69, 193213.

BrinkLey, L., BASEHOAR, G., and AVERY, J. (1978). Effects of craniofacial structures on mouse palatal closure in vitro. J. Dent. Res. 57 , 402-411. 
Brinkley, L., BaSehoar, G., Branch, A., and Avery, J. (1975). A new in vitro system for studying secondary palatal development. J. Embryol. Exp. Morphol 34, 485-495.

DIEWERT, V. M. (1978). A quantitative coronal plane evaluation of craniofacial growth and spatial relations during secondary palate development in the rat. Arch. Oral Biol. 23, 607-629.

Ferguson, M. W. J. (1977). The mechanism of palatal shelf elevation and the pathogenesis of cleft palate. Virchows Arch A: Pathol Anut. Histol. 375, 97-113.

Hudson, C., and ShapIRo, B. (1973). A radioautographic study of deoxyribonucleic acid synthesis in embryonic rat palatal shelf epithelium with reference to the concept of programmed cell death. Arch. Oral Biol. 18, 77-84.

KochHAR, D., and JoHnSON, E. (1965). Morphologic and autoradiographic studies of cleft palate induced in rats by maternal hypervitaminosis A. J. Embryol. Exp. Morphol. 14, 223-238.

Lewis, C., Thibault, L., Pratt, R., and Brinkley, L. (1980). An im- proved culture system for secondary palatal elevation. In Vitro 16, 453-460.

POURTOIS, M. (1972). Morphogenesis of the primary and secondary palate. In "Developmental Aspects of Oral Biology" (H. C. Slavkin and L. A. Bavetta, eds.), pp. 81-108. Academic Press, London.

SCHEFFÉ, H. 1959. "Analysis of Variance." Wiley, New York.

SWENEY, L. R., and SHAPIRO, B. L. (1970). Histogenesis of Swiss White Mouse secondary palate from nine and one-half days to fifteen and one-half days in utero. I. Epithelial-mesenchymal relationshipslight and electron microscopy. J. Morphol. 130, 435-450.

Walker, B. E., and Fraser, C. F. (1956). Closure of the secondary palate in three strains of mice. $J$. Embryol. Exp. Morphol 4, 176189.

WALKER, B. E., and B. CRAIN, JR., (1960). Effects of hypervitaminosis A on palate development in two strains of mice. Amer. J. Anat. 107, 49-58. 